\title{
Historias Gineco-Obstétricas Precodificadas
}

\author{
Universidad de Caldas - Facultad de Medicina \\ Hospital Universitario de Caldas \\ Departamento de Ginecología y Obstetricia
}

Dres.: Mario Calle Mesa* y Carlos Alberto Esparza Duque**

\section{Introducción}

Con la experiencia adquirida en el manejo de formularios precodificados, en estudios cooperativos y prospectivos realizados por el Departamento de Ginecología y Obstetricia de la Universidad de Caldas y el Programa Regional de Investigaciones en Fecundidad (PRIF) de la CCRP, nos propusimos diseñar varios modelos de historias ginecoobstétricas para obtener información sobre diversos aspectos importantes de la especialidad, tomando como punto de referencia algunos del Programa Internacional de Investigaciones en Fecundidad (IPRF).

EI propósito fundamental de este estudio es valorar nuevos instrumentos para el registro de información clínica y determinar su eficiencia, ventajas, desventajas, etc., en relación con las historias clínicas vigentes.

Estas últimas, adolecen de serias deficiencias que inevitablemente inciden

\footnotetext{
* Jefe Dpto. de Gineco-Obstetricia.

** Profesor Asociado.

Departamento Gineco-Obstetricia.
}

en forma desfavorable sobre la calidad de la asistencia, docencia e investigación que se realizan con base en ellas.

Es necesario reformar a la luz de los conocimientos actuales sobre información el sistema de registros hospitalarios si se desea progresar eficazmente en estos campos.

Toda la información registrada puede procesarse en computadora, lo que permite conocer rápidamente los resultados y facilita el análisis de un inmenso número de variables consignadas en ella.

Tenemos especial interés en proponer el empleo de estos modelos de Historias Gineco-Obstétricas a través de FECOLSOG y del MINISTERIO DE SALUD en los diferentes hospitales del país.

\section{Material y Métodos}

En 1979, empezamos a diseñar las historias para registro de PARTO y PUERPERIO y de ABORTO, haciéndole algunas modificaciones a los modelos empleados por el PROGRAMA REGIONAL DE INVESTIGACIONES EN FECUNDIDAD (PRIF). 
En 1980, elaboramos diez formularios originales para consignar información sobre los siguientes aspectos: Atención prenatal, Partograma, Recién nacido, Fórceps, Cesárea, Embarazo ectópico, Neoplasia trofoblástica, Historia ginecológica, Endocrınología y esterilidad y Patología mamaria.

Se contó con la eficaz colaboración de la División de Arquitectura del Servicio de Salud de Caldas para el diseño original de las historias y de la Universidad de Caldas para la impresión de las mismas.

La mayoría de las historias están configuradas en una sola hoja y contienen una amplísima información sobre características socio demográficas de las pacientes, antecedentes familiares, personales y gineco-obstétricas, información médica, examen clínico, diagnósticos presuntivos y definitivos precodificados en algunas de ellas, así como también tratamiento y seguimiento en otras.

Las variables y códigos previamente definidos permiten registrar la información con criterios unificados en forma rápida y de fácil ejecución.

Los doce modelos de historias precodificadas se están utilizando en el Departamento de Ginecología y Obstetricia de la Universidad de Caldas, desde el 10. de julio de 1980.

\section{Resultados}

Se presentan apartes de algunos de los modelos.

En el período de un año, comprendido entre el 10. de julio de 1980 y el 30 de junio de 1981, se han diligenciado los siguientes formularios:

\footnotetext{
* 6 meses.
}

REgISTROS PRECODIFICADOS DILIGENCIADOS

EN UN AÑO (1980 - 1981)

\begin{tabular}{|lrrl|}
\hline Registro & Número & Variables & Códigos \\
\hline Atención prenatal & 436 & 25 & Más de 51 \\
Parto y puerperio & 3.211 & 51 & Más de 171 \\
Recién nacido & 3.371 & 38 & Más de 141 \\
Fórceps y ventosa obstétrica & 109 & 16 & Más de 83 \\
Cesárea & 448 & 25 & Más de 56 \\
Aborto & 621 & 52 & Más de 176 \\
Embarazo ectópico & 56 & 46 & Más de 83 \\
Historia ginecológica & 388 & 46 & Más de 200 \\
Endocrinología y & 56 & & Más de 300 \\
esterilidad* & & & \\
\hline Total & 8.696 & - & - \\
\hline
\end{tabular}

Esperamos aprovechar la enorme información consignada en estas historias, con el propósito de realizar nuevos estudios.

\section{Comentarios}

La experiencia obtenida con el empleo de diferentes modelos de Historias Gineco-Obstétricas precodificadas ren nuestro Departamento, corrobora la inquietud que de tiempo atrás teníamos sobre la necesidad de cambiar las historias clínicas vigentes, por otras más eficaces y de fácil ejecución, que permitan consignar la más variada información con fines asistenciales, docentes e investigativos.

No podemos continuar indiferentes ante el acelerado desarrollo de la tecnología computarizada, aplicada profusamente en los registros hospitalarios, de muchos países.

Se ha conseguido buena aceptación por parte de estudiantes, internos $y$ residentes, para el correcto diligenciamiento de las historias.

Nos proponemos corregir las deficiencias que presentan algunos de los modelos, para elaborar los instructivos correspondientes. 


\title{
Resumen y Conclusiones
}

Se presentan doce modelos de Historias Gineco-Obstétricas precodificadas que se vienen empleando en el Departamento de Ginecología y Obstetricia de la Universidad de Caldas, desde el 10. de julio de 1980.

En otro estudio se presentan los resultados conseguidos con este valioso instrumento de registro hospitalario.

Proponemos a FECOLSOG y al MI-
NISTERIO DE SALUD, un análisis de estos formularios para su empleo en la mayoría de los hospitales del país, con el fin de lograr los objetivos que no se consiguen satisfactoriamente con las historias empleadas en nuestro medio.

Cuando se disponga de historias unificadas, podremos realizar estudios prospectivos y cooperativos que permitan conocer nuestra propia patología, y comparar los resultados.

\section{PRECODED OB-GYN PATIENT RECORDS}

\section{Summary and conclusions}

This article presents 12 models of Ob-gyn patient records with a precoded design, used at the Obstetrics and Gynecology Department of University of Caldas since july 1, 1980.

A separate study presents the results obtained through the utilization of this important hospital record system.

The authors address the Colombian Federation of Societies of Obstetrics and
Gynecology (FECOLSOG) and the Ministry of Health, so that these institutions proceed to conduct a deep analysis of these forms in order to improve the data and record system of hospitals and clinics in Colombia.

The use of unified medical records will favor prospective and cooperative studies which will provide better knowledge about diverse pathologies and facilitate comparison.

Quiénes estén interesados en los modelos, propuestos por los autores de este artículo, de historias gineco-obstétricas precodificadas, rogamos dirigirse a los Dres.:

\author{
Mario Calle Mesa y Carlos Alberto Esparza Duque \\ Departamento de Obstetricia y Ginecología \\ Hospital Universitario de Caldas \\ Manizales, Colombia.
}

Los modelos incluidos en el presente artículo son:

Atención prenatal

Parto y puerperio

Recién nacido

Cesárea

Aborto

Fórceps y ventosa obstétrica
Embarazo ectópico

Neoplasia trofebiestica

Historia Ginecológica

Endocrinología - esterilidad

Patología mamaria 


\section{Referencias}

1. MELENDEZ, L. J: Historias Clínicas Funcionales. De Universitas Médica 10:62, 1968.

2. Conceptos Generales, Administración de Registros Médicos y Modelo de Historia Clínica Básica. Ministerio de Salud. Oficina de Planeación. División de Información, 1978.

3. WEED LL: Medical Records that Guide and Teach. New Eng J Med 278: 652, 1968.
4. WEED LL: Physicians of the Future. $\mathrm{N}$ Eng J Med 304: 903, 1981.

5. WEED LL: New Premises and New Tools for Medical Education. Unpublished.

6. Atención del Parto Hospitalario en Colombia. Primeros Hallazgos. Primera Edición. Corporación Centro Regional de Población. Bogotá, 1979. pp. 23-52. 